\title{
Understanding the evolutionary potential of epigenetic variation: a comparison of heritable phenotypic variation in epiRILs, RILs, and natural ecotypes of Arabidopsis thaliana
}

\author{
Yuan-Ye Zhang ${ }^{1,2} \cdot$ Vit Latzel $^{1,3} \cdot$ Markus Fischer $^{1} \cdot$ Oliver Bossdorf ${ }^{1,4}$ \\ Received: 15 November 2017 / Revised: 8 May 2018 / Accepted: 16 May 2018 / Published online: 6 June 2018 \\ (c) The Genetics Society 2018
}

\begin{abstract}
Increasing evidence for epigenetic variation within and among natural plant populations has led to much speculation about its role in the evolution of plant phenotypes. However, we still have a very limited understanding of the evolutionary potential of epigenetic variation, in particular in comparison to DNA sequence-based variation. To address this question, we compared the magnitudes of heritable phenotypic variation in epigenetic recombinant inbred lines (epiRILs) of Arabidopsis thaliana-lines that mainly differ in DNA methylation but only very little in DNA sequence-with other types of $A$. thaliana lines that differ strongly also in DNA sequence. We grew subsets of two epiRIL populations with subsets of two genetic RIL populations, of natural ecotype collections, and of lines from a natural population in a common environment and assessed their heritable variation in growth, phenology, and fitness. Among-line phenotypic variation and broad-sense heritabilities tended to be largest in natural ecotypes, but for some traits the variation among epiRILs was comparable to that among RILs and natural ecotypes. Within-line phenotypic variation was generally similar in epiRILs, RILs, and ecotypes. Provided that phenotypic variation in epiRILs is mainly caused by epigenetic differences, whereas in RILs and natural lines it is largely driven by sequence variation, our results indicate that epigenetic variation has the potential to create phenotypic variation that is stable and substantial, and thus of evolutionary significance.
\end{abstract}

\section{Introduction}

It is common textbook knowledge that heritable phenotypic variation is the raw material for evolution, and that this phenotypic variation results from underlying variation in

Electronic supplementary material The online version of this article (https://doi.org/10.1038/s41437-018-0095-9) contains supplementary material, which is available to authorized users.

Yuan-Ye Zhang

zhangyuanye@xmu.edu.cn

1 Institute of Plant Sciences, University of Bern, CH-3013 Bern, Switzerland

2 Key Laboratory of the Ministry of Education for Coastal and Wetland Ecosystems, College of the Environment and Ecology, Xiamen University, Xiamen, Fujian 361102, China

3 Institute of Botany of the ASCR, CZ-252 43 Průhonice, Czech Republic

4 Plant Evolutionary Ecology, University of Tübingen, D-72076 Tübingen, Germany
DNA sequence (Fisher 1930; Falconer and Mackay 1996; Futuyma 2013). Recent research changes this view, as there is now increasing evidence that heritable phenotypic variation can also be created by variation in epigenetic modifications such as the methylation of cytosines in the DNA, or various histone modifications (Richards 2006; Bird 2007; Cortijo et al. 2014; Kooke et al. 2015; Morgado et al. 2017; Richards et al. 2017). Current research focuses particularly on DNA methylation, and has shown that in plants variation in DNA methylation is partly transmitted across generations (Becker et al. 2011; Schmitz et al. 2011; van der Graaf et al. 2015), and that intra-population and inter-population variation in DNA methylation is common in natural plant populations (Schmitz et al. 2013b; Kawakatsu et al. 2016; Vidalis et al. 2016). Moreover, epigenetic variation can cause heritable trait variation, and it can be associated with phenotypic or environmental variation in nature (Herrera and Bazaga 2011; Zhang et al. 2013; Wilschut et al. 2016). Together, this suggests that the study of evolution and adaptation may be incomplete without taking heritable epigenetic variation into account. 
However, in spite of this increasing evidence for natural epigenetic variation and its phenotypic consequences in natural and experimental populations (Cubas et al. 1999; Cortijo et al. 2014; Kooke et al. 2015; Kawakatsu et al. 2016; Wilschut et al. 2016), we are still far from understanding the evolutionary potential of epigenetic variation. This is mainly because of the difficulty of disentangling epigenetic from DNA sequence-based effects in natural systems (Richards 2006; Bossdorf et al. 2008). A rare opportunity for testing the phenotypic consequences of epigenetic variation in a relatively straightforward, albeit artificial, fashion is provided by epigenetic recombinant inbred lines (epiRILs) of Arabidopsis thaliana (Johannes et al. 2009; Reinders and Paszkowski 2009; Reinders et al. 2009). EpiRILs are highly variable at the level of DNA methylation, but harbor only very little DNA sequence variation. The creation of epiRILs is similar to the creation of classic recombinant inbred lines (RILs), where two genetically divergent parents are crossed, and inbred lines are established from their recombinant offspring. In epiRILs, the two parents are nearly identical in DNA sequence, but-because of mutations in their DNA methylation machinery-differ strongly in their patterns and overall degree of DNA methylation (Johannes et al. 2009; Reinders and Paszkowski 2009; Reinders et al. 2009), and the derived epiRILs represent different mosaics of their parents' epigenomes.

Currently, two populations of $A$. thaliana epiRILs exist. Both have been created by crossing a hypomethylation mutant of the Col-0 ecotype (metl or $d d m 1$; see 'Materials and methods' for more details) to its near-isogenic and normally methylated wild-type. F2 or backcross homozygous at MET1 or DDM1 respectively, were then selected and propagated through six generations of selfing. Molecular analyses confirmed that a substantial fraction of the DNA methylation variation created through recombination was inherited in a Mendelian manner (Colome-Tatche et al. 2012). Moreover, experimental studies showed that the epiRILs also harbored significant phenotypic variation in plant morphology, growth rate, and responses to biotic and abiotic stress (Johannes et al. 2009; Roux et al. 2011; Latzel et al. 2012; Zhang et al. 2013; Kooke et al. 2015), and that this phenotypic variation constituted evolutionary potential (Zhang et al. 2013) as well as functional intraspecific diversity (Latzel et al. 2013). A detailed epiQTL mapping analysis of the $d d m 1$ epiRIL population showed that, although the lines contained a small amount of DNA sequence variation, a large (60-90\%) part of the heritability of flowering time and root length was associated with differential DNA methylation but not DNA sequence variation (Cortijo et al. 2014).

However, while these previous studies together provided some indication that heritable epigenetic variation can be ecologically and evolutionarily relevant, we are still far from understanding the real-world significance of epigenetically-based phenotypic variation. To put the phenotypic variation in epiRILs into context, a useful next step would be to directly compare the magnitude of phenotypic variation in epiRILs - representing the upper limit of what can be achieved through DNA methylation-to systems that are (presumably) mainly driven by DNA sequence variation, such as the variation within and among natural populations, or genetic RILs. As estimates of phenotypic variation are strongly influenced by the environment in which they are made (Simons and Roff 1994; Conner et al. 2003; Hansen et al. 2011), it is imperative that such comparisons are carried out under identical environmental conditions.

One previous study already compared the phenotypic variation of $d d m 1$-derived epiRILs to that of some natural ecotypes and found that epiRILs generally had a similar heritability structure across different traits as the natural ecotypes, but that the magnitude of phenotypic variation was significantly lower in the epiRILs (Roux et al. 2011). Here, we built on this study to attempt a more comprehensive comparison of epiRILs with different types of $A$. thaliana lines that are either representing natural variation, or that are frequently used for studying genetically-based phenotypic variation. Specifically, we studied subsets of two different epiRIL populations alongside equal-sized subsets of two genetic RIL populations, two natural ecotype collections from across Eurasia and North America, and of lines within a natural population. While the latter two categories represent natural variation shaped by evolutionary forces at geographic and local scales, respectively, genetic RILs and epiRILs resulted from artificial crosses and thus represent the upper limits of heritable variation which can be achieved by such means. It is important to stress that none of the studied systems is pure in the sense that there is only variation at the level of DNA methylation or DNA sequence, but that they all harbor genetic and epigenetic variation, albeit in very different relative magnitudes. In epiRILs, there is large epigenetic variation but very little sequence variation, whereas in natural ecotypes and RILs there is large genetic and epigenetic variation, but much of the epigenetic variation is likely under genetic control (Schmitz et al. 2013a; Dubin et al. 2015). Thus, the key assumption of our study is that in epiRILs phenotypic variation is mainly caused by epigenetic differences (as shown by Cortijo et al. 2014), whereas in RILs and natural ecotypes it is primarily driven by sequence differences. Based on this assumption, we ask the following question: how does the heritable phenotypic variation within and among epiRILs compare to the variation in genetic RILs and natural ecotypes, when studied under identical environmental conditions? 
Table 1 Trait means $(\bar{X})$ and variances for all studied groups of $A$. thaliana lines $\left(\sigma_{L}^{2}=\right.$ among lines, $\sigma_{B}^{2}=$ among blocks, $\sigma_{E}^{2}=$ within lines $=$ residuals)

\begin{tabular}{|c|c|c|c|c|c|c|}
\hline & $\bar{X}$ & $\sigma_{L}^{2}$ & $\sigma_{B}^{2}$ & $\sigma_{E}^{2}$ & $\begin{array}{l}\sigma_{L}^{2} \text { group vs. } d d m 1- \\
\text { epiRILs }(P)\end{array}$ & $\begin{array}{l}\sigma_{L}^{2} \text { group vs. met1- } \\
\text { epiRILs }(P)\end{array}$ \\
\hline \multicolumn{7}{|c|}{ Flowering time (days) } \\
\hline epiRILs $d d m 1$ & 27.76 & $3.76^{* * *}$ & $0.52^{*}$ & 8.28 & - & - \\
\hline epiRILs met1 & 31.12 & $14.01^{* * *}$ & $1.77^{* *}$ & 12.70 & 0.001 & - \\
\hline RILs Ler $\times$ Col & 28.18 & $13.13^{* * *}$ & $0.00^{\mathrm{NS}}$ & 11.00 & 0.002 & 0.851 \\
\hline RILs Bay $\times$ Sha & 26.19 & $7.71^{* * *}$ & $0.65^{*}$ & 7.89 & 0.059 & 0.112 \\
\hline Local population & 26.93 & $2.43^{* *}$ & $0.00^{\mathrm{NS}}$ & 7.52 & 0.272 & $<0.001$ \\
\hline Ecotypes NA & 32.35 & $13.14^{* * * *}$ & $1.46^{*}$ & 9.34 & 0.002 & 0.857 \\
\hline Ecotypes EU & 29.11 & $17.25^{* * *}$ & $0.00^{\mathrm{NS}}$ & 6.41 & $<0.001$ & 0.573 \\
\hline \multicolumn{7}{|l|}{ Stem number } \\
\hline epiRILs $d d m 1$ & 4.43 & 0.00 & $0.04^{\mathrm{NS}}$ & 1.67 & - & - \\
\hline epiRILs met 1 & 3.80 & $0.87^{* *}$ & $0.01^{\mathrm{NS}}$ & 1.95 & $<0.001$ & - \\
\hline RILs Ler $\times$ Col & 3.25 & $1.08^{* * *}$ & $0.00^{\mathrm{NS}}$ & 1.30 & $<0.001$ & 0.590 \\
\hline RILs Bay $\times$ Sha & 4.38 & $0.58^{*}$ & $0.25^{* *}$ & 2.45 & $<0.001$ & 0.281 \\
\hline Local population & 4.25 & $0.52^{* *}$ & $0.14^{*}$ & 1.74 & $<0.001$ & 0.177 \\
\hline Ecotypes NA & 3.94 & $1.39^{* * * *}$ & $0.16^{*}$ & 2.27 & $<0.001$ & 0.228 \\
\hline Ecotypes EU & 3.63 & $5.65^{* * *}$ & $0.16^{*}$ & 2.04 & $<0.001$ & $<0.001$ \\
\hline \multicolumn{7}{|l|}{ Plant height $(\mathrm{cm})$} \\
\hline epiRILs $d d m 1$ & 20.43 & $1.38^{*}$ & $0.53^{*}$ & 5.35 & - & - \\
\hline epiRILs met1 & 20.34 & $22.20^{* * *}$ & $0.00^{\mathrm{NS}}$ & 13.26 & $<0.001$ & - \\
\hline RILs Ler $\times$ Col & 17.38 & $16.44^{* * *}$ & $0.98^{* *}$ & 4.50 & $<0.001$ & 0.450 \\
\hline RILs Bay $\times$ Sha & 19.34 & $6.60^{* * *}$ & $2.84^{* * *}$ & 9.42 & $<0.001$ & 0.002 \\
\hline Local population & 19.41 & $11.52^{* * *}$ & $2.64^{* * *}$ & 6.01 & $<0.001$ & 0.095 \\
\hline Ecotypes NA & 19.20 & $46.17^{* * * *}$ & $0.39^{\mathrm{NS}}$ & 11.73 & $<0.001$ & 0.058 \\
\hline Ecotypes EU & 16.23 & $105.0^{* * * * *} 8$ & $0.00^{\mathrm{NS}}$ & 17.57 & $<0.001$ & $<0.001$ \\
\hline \multicolumn{7}{|l|}{ Fruit number } \\
\hline epiRILs $d d m 1$ & 101.30 & $374.1^{* *}$ & $373.0^{* * *}$ & 1224.7 & - & - \\
\hline epiRILs met1 & 58.69 & $976.8^{* * *}$ & $133.2^{*}$ & 1314.2 & 0.011 & - \\
\hline RILs Ler $\times$ Col & 71.98 & $477.5^{* * * *}$ & $77.4^{\mathrm{NS}}$ & 788.9 & 0.540 & 0.077 \\
\hline RILs Bay $\times$ Sha & 87.43 & $909.5^{* * * *}$ & $421.3^{* * * *}$ & 1083.1 & 0.020 & 0.846 \\
\hline Local population & 91.00 & $965.6^{* * *}$ & $288.9^{* * *}$ & 1315.5 & 0.018 & 0.961 \\
\hline Ecotypes NA & 65.38 & $1514.6^{* * *}$ & $292.0^{* *}$ & 1371.0 & $<0.001$ & 0.254 \\
\hline Ecotypes EU & 84.59 & $4750.0^{* * *}$ & $144.8^{* *}$ & 1396.4 & $<0.001$ & $<0.001$ \\
\hline \multicolumn{7}{|c|}{ Rosette diameter $(\mathrm{mm})$} \\
\hline epiRILs $d d m 1$ & 32.99 & $9.66^{*}$ & $10.8^{* * * *}$ & 42.2 & - & - \\
\hline epiRILs met1 & 54.90 & $1086.6^{* * *}$ & $16.5^{* * *}$ & 66.6 & $<0.001$ & - \\
\hline RILs Ler $\times$ Col & 26.24 & $39.7^{* * *}$ & $10.3^{* * *}$ & 23.4 & 0.001 & $<0.001$ \\
\hline RILs Bay $\times$ Sha & 27.36 & $322.9^{* * *}$ & $20.0^{* * *}$ & 41.1 & $<0.001$ & 0.002 \\
\hline Local population & 32.39 & $73.3^{* * *}$ & $29.2^{* * * *}$ & 47.5 & $<0.001$ & $<0.001$ \\
\hline Ecotypes NA & 75.78 & $1549.7^{* * *}$ & $50.0^{* * *}$ & 196.3 & $<0.001$ & 0.354 \\
\hline Ecotypes EU & 73.11 & $2669.0^{* * *}$ & $23.4^{* * *}$ & 139.4 & $<0.001$ & 0.016 \\
\hline \multicolumn{7}{|c|}{ Aboveground biomass (mg) } \\
\hline epiRILs $d d m 1$ & 142.45 & $568.5^{*}$ & $606.7^{* * * *}$ & 2583.3 & - & - \\
\hline epiRILs met1 & 186.91 & $12960.4^{* * *}$ & $419.4^{*}$ & 5322.8 & $<0.001$ & - \\
\hline RILs Ler $\times$ Col & 87.02 & $812.4^{* * *}$ & $153.1^{*}$ & 1004.1 & 0.377 & $<0.001$ \\
\hline RILs Bay $\times$ Sha & 125.80 & $5001.0^{* * * *}$ & $593.4^{* * * *}$ & 2208.1 & $<0.001$ & 0.012 \\
\hline
\end{tabular}


Table 1 (continued)

\begin{tabular}{|c|c|c|c|c|c|c|}
\hline & $\bar{X}$ & $\sigma_{L}^{2}$ & $\sigma_{B}^{2}$ & $\sigma_{E}^{2}$ & $\begin{array}{l}\sigma_{L}^{2} \text { group vs. } d d m 1- \\
\text { epiRILs }(P)\end{array}$ & $\begin{array}{l}\sigma_{L}^{2} \text { group vs. met1- } \\
\text { epiRILs }(P)\end{array}$ \\
\hline Local population & 132.88 & $4431.5^{* * *}$ & $730.7^{* * *}$ & 2481.4 & $<0.001$ & 0.007 \\
\hline Ecotypes NA & 312.82 & $29597.1^{* * *}$ & $1449.7^{*}$ & 11127.0 & $<0.001$ & 0.033 \\
\hline Ecotypes EU & 295.14 & $36315.4^{* * * *}$ & $275.5^{\mathrm{NS}}$ & 8624.3 & $<0.001$ & 0.006 \\
\hline
\end{tabular}

Significance levels of variance components are indicated by asterisks $(* * * P<0.001, * * P<0.01, * P<0.05)$. The last two columns give the probability ( $P$-values) that variation among lines $\left(\sigma_{L}^{2}\right)$ of a group is equal to that of $d d m 1$ - and met 1 -derived epiRILs, respectively.

\section{Materials and methods}

\section{Plant materials}

We worked with two populations of epiRILs of Arabidopsis thaliana, which are established from the cross between Col0 and its methylation mutant $d d m l$ (Johannes et al. 2009) and metl (Reinders et al. 2009) respectively. Both are point mutants that affect the maintenance of DNA methylation (see Kakutani et al. 1995, for $d d m l$ and Finnegan and Kovac 2000, for metl) and that result in $\sim 70 \%$ genomewide loss of DNA methylation compared to the wild-type. In addition to the two epiRIL populations, we also included two populations of classic RILs, Ler $\times$ Col (Lister and Dean 1993), a cross between the two most important lab lines of A. thaliana, and Bay $\times$ Sha (Loudet et al. 2002), a cross between the geographically and ecologically distinct natural ecotypes Bay-0 (Bayreuth, Germany), and Sha (Shahdara, Tajikistan). Both populations have been used widely for QTL analyses (Alonso-Blanco and Koornneef 2000; Koornneef et al. 2004). From each of the two epiRIL and two RIL populations, we randomly selected around 30 lines for the phenotype screening. In addition, we included 23 lines from a local population in Europe (Bu; Burghaun, Germany). Finally, we also included broad-scale geographic collections of natural ecotypes from Eurasia (31 lines) and North America (25 lines). The ecotypes were selected randomly from those available at the stock center, with the restriction that they should come from distinct geographic locations. For North American ecotypes, this limited the number of lines to 25 . Seeds of $d d m 1$-epiRILs and met 1 epiRILs were provided by the labs of Vincent Colot and Jurek Paszkowski, respectively. All other seeds were obtained from the Arabidopsis stock center.

\section{Phenotyping}

For each of the 199 lines used, we cold-dark-stratified seeds in petri dishes with moist filter paper at $4{ }^{\circ} \mathrm{C}$ for one week. After that, we pipetted about 4-5 seeds of each line into 0.3$\mathrm{L}$ pots filled with a standard potting soil. Approximately five days after germination we thinned all seedlings to one per pot. The pots were placed in an unheated greenhouse in Bern, Switzerland, in late spring 2011. We did not use any additional lighting, so that the plants experienced light conditions (day lengths and light intensities) that are typical for spring, the most common growing season of A. thaliana. We arranged the pots in five spatial blocks that contained one replicate of each line. Thus, there were five replicates per line in total. Within each spatial block the pots were randomized. The plants were regularly bottom-watered by flooding tables with water containing $500 \mathrm{ppm}$ of a $18-12$ 18 NPK fertilizer. For each plant, we recorded flowering time as the number of days from planting to the first open flower. Eight weeks after sowing we recorded the maximum height, rosette diameter, number of stems, and number of siliques of each plant. We harvested all aboveground biomass, dried it at $70^{\circ}$ for $48 \mathrm{~h}$ and weighed it. 10 RILs from the Ler $\times$ Col population did not germinate well enough to be included in the study, so the final data set included only 189 lines (see Supplementary Table S1 for a list of the specific lines included).

\section{Statistical analyses}

In our experiment, the total variance $\left(\sigma_{T}^{2}\right)$ of a trait can be partitioned into the variance among lines $\left(\sigma_{L}^{2}\right)$, the variance among blocks $\left(\sigma_{B}^{2}\right)$, and the residual (or within-line) variance $\left(\sigma_{E}^{2}\right)$. For each of the six measured phenotypic traits, we fitted a mixed-effects model using the nlme package (Pinheiro et al. 2013) in $\mathrm{R}$ version 3.0.2 (R Core Team 2013), with block as fixed effect and line as random effect. We tested the significance of line effects and block effects using likelihood ratio test where the full model was tested against the reduced model excluding the tested factor. For each trait and each group of lines, we also estimated broadsense heritability as the proportion of the total variance attributed to variance among lines $\left(H^{2}=\sigma_{L}^{2} / \sigma_{T}^{2}\right)$, and the among-line and within-line coefficients of variation as the ratio between the standard deviations of the respective effects and their means $\left(C V_{L}=\sigma_{L} / \overline{\mathrm{X}}\right.$ and $\left.C V_{E}=\sigma_{e} / \overline{\mathrm{X}}\right)$. We obtained standard errors for these measures from 1000 bootstrap samples, and we then used $F$-test to test the 


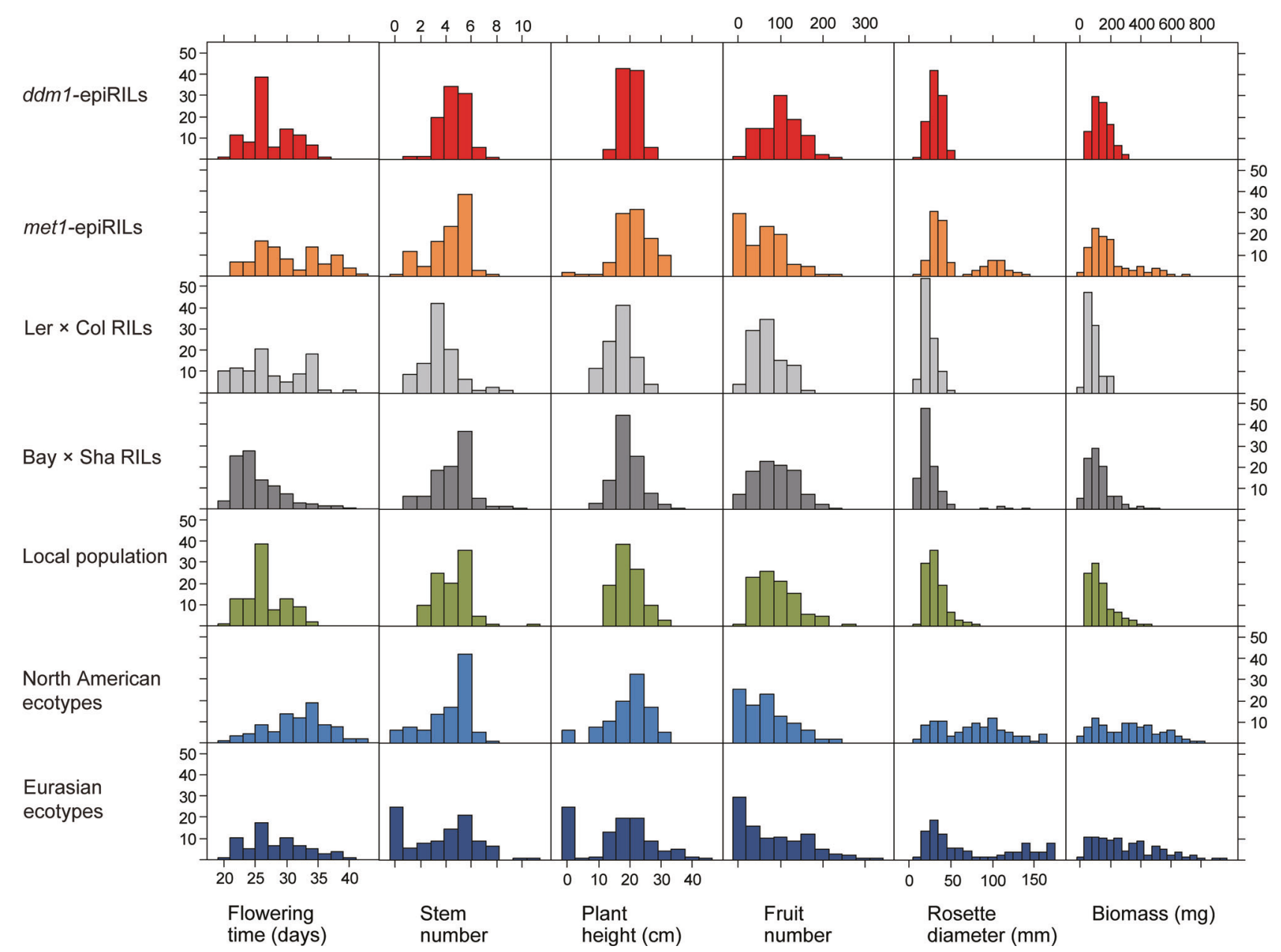

Fig. 1 Distributions of phenotypes within different groups of Arabidopsis thaliana $(\mathrm{RILs}=$ genetic recombinant inbred lines; epiRILs = epigenetic recombinant inbred lines), for six different phenotypic traits, when compared in a common greenhouse environment. Plants with large rosette diameter (in met1-epiRILs, Bay $\times$ Sha RILs, and ecotypes) are late-flowering, whereas plants with zero height and number of fruits are individuals that did not bolt or flower during the experiment, possibly representing a winter-annual strategy. The geographic (Eurasian or North American) collections of ecotypes are in blue, the local European population in green, RILs in grey, and epiRILs in orange

probability that the $\sigma_{L}^{2}$ of epiRILs was equal to that of other groups.

\section{Results}

For almost all traits measured, there was significant amongline differentiation in phenotypes, as indicated by the among-line variance components in both epiRIL populations as well as in all other groups (Table 1, Figs. 1 and 2). The lowest values for among-line variation were in the $d d m 1$-epiRILs, the largest in the Eurasian ecotype collections, and all other groups had intermediate levels of among-line variation. In contrast to the $d d m l$-epiRILs, the among-line variation of metl-epiRILs was similar to that of RILs, the local population, and the North American ecotypes. Broad-sense heritability estimates in $d d m 1$-epiRILs ranged from 0.18 to 0.31 , whereas in metl-
epiRILs heritability estimates for the same traits were higher and ranged from 0.37 to 0.92 . The heritability estimates for the two epiRIL populations, and their differences to other groups, closely followed the among-line variances, as within-line variances, estimated by the coefficient of variation of the residuals $\left(C V_{E}\right)$, were rather constant across groups (Fig. 2). Interestingly, the met1epiRILs appeared to harbor several distinct life-history types: early-flowering lines which reproduced quickly, later-flowering lines which grew larger rosettes before they flowered, and lines which did not flower at all during our experiment (Fig. 1 and Supplementary Figure S1). Besides the metl-epiRILs, the three life-history types were also frequent among natural ecotypes from Europe and North America, but there was only one late-flowering Bay $\times$ Sha line, and none at all among Ler $\times$ Col RILs, $d d m 1$-epiRILs or in the local population (Fig. 1 and Supplementary Figure S1). 


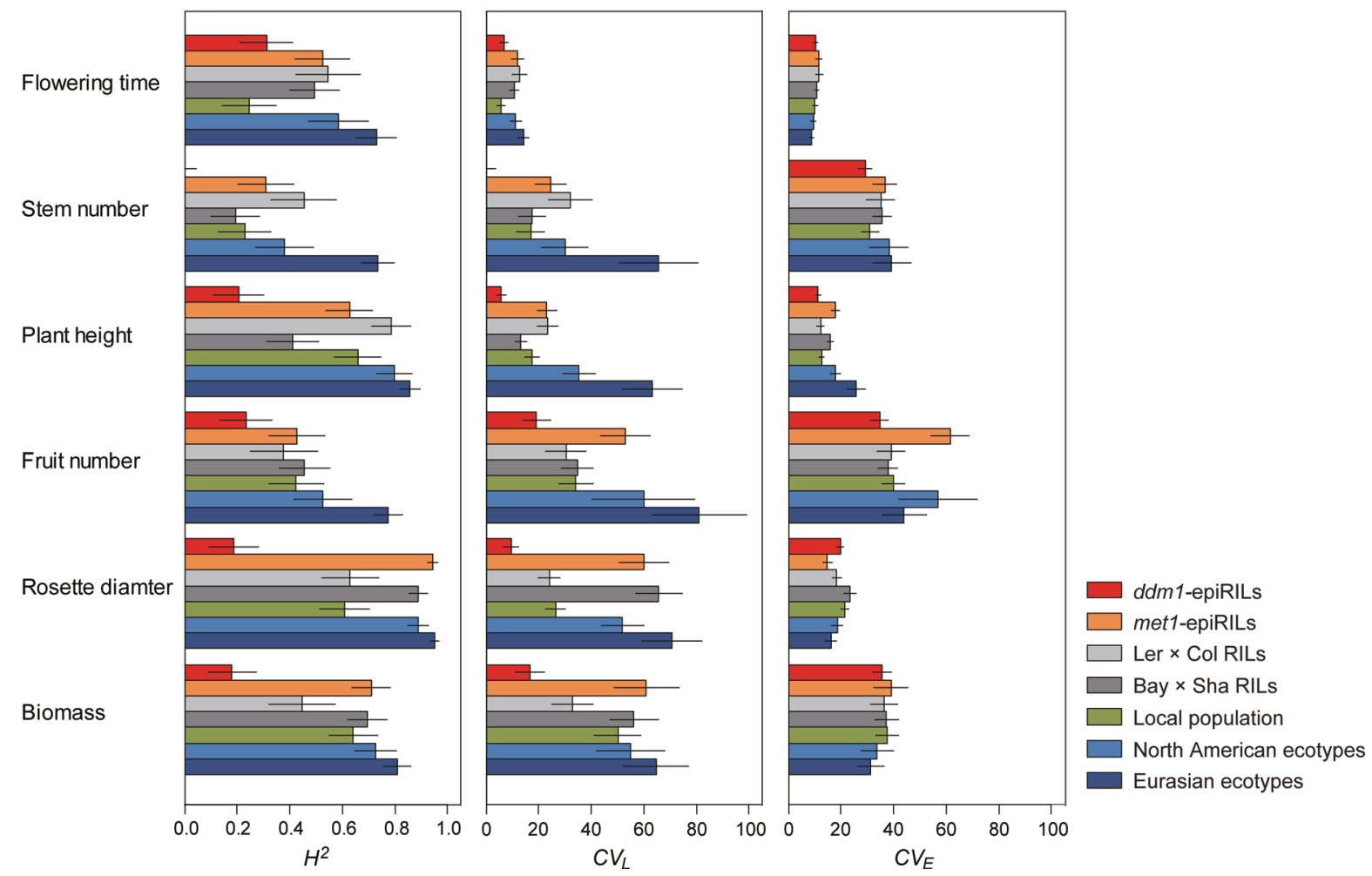

Fig. 2 Estimates of among-line and within-line phenotypic variation for all groups of A. thaliana lines studied. Among-line variance is estimated by broad-sense heritabilities $\left(H^{2}\right)$ as well as among-line coefficients of variation $\left(C V_{L}\right)$. Within-line variation is estimated by the residual coefficient of variation $\left(C V_{E}\right)$. The geographic (Eurasian or North American) collections of ecotypes are in blue, the local European population in green, RILs in grey, and epiRILs in orange

\section{Discussion}

Epigenetic variation can create heritable phenotypic variation in plants, but so far we do not have a good understanding of the significance of such epigenetically-based variation, in particular in comparison to genetically-based variation. Here, we directly compared the phenotypic variation within and among epiRILs of A. thaliana to that of natural ecotypes and genetic RILs under identical environmental conditions. We found that the magnitude and stability of heritable phenotypic variation in epiRILs was comparable to that in RILs and natural ecotypes. Under the assumption that the phenotypic variation in epiRILs is mainly caused by epigenetic differences, whereas in RILs and natural ecotypes it is mainly driven by sequence variation, our results indicate that in principle variation in DNA methylation can create heritable phenotypic variation that is substantial and of a similar order of magnitude as that observed in natural and more DNA sequence-based systems.

Levels of within-line variation were similar across all studied genetic and epigenetic lines. In general, within-line variation in phenotype may reflect environmental or developmental stochasticity, or it can be created by spontaneous mutation or epimutation. We would have expected within-line phenotypic variability to be highest in epiRILs because TEs are activated in these lines, and epigenetic inheritance is less stable than the inheritance of DNA sequence differences (Becker et al. 2011; Schmitz et al. 2011; Cortijo et al. 2014; van der Graaf et al. 2015). However, our results suggest that the effects of TE insertions and spontaneous epigenetic changes may often not propagate to the phenotypic level, probably because many of them are neutral (Ossowski et al. 2010; Becker et al. 2011; Schmitz et al. 2011) or have minor phenotypic consequences.

While within-line variation was similar in all groups, among-line variation was more variable. The heritability estimates of $d d m l$-epiRILs were comparable to previous studies of the same epiRIL population (Johannes et al. 2008; Roux et al. 2011). North American ecotypes generally tended to be less variable than Eurasian ecotypes, a consequence of their introduction history (Platt et al. 2010). A. thaliana was introduced to North America only 150-200 years ago. Moreover, the low variability of flowering time within the natural population is consistent with previous findings (Brachi et al. 2013; Wolfe and Tonsor 2014) and is likely due to stabilizing selection at the local scale. While some of the patterns revealed in our study are consistent with previous observations comparing either epiRILs or 
RILs to ecotypes (Ungerer et al. 2002; Roux et al. 2011), we are not aware of any previous study that conducted such a broad comparison of epiRILs, RILs and natural ecotypes as in our study.

We found that among-line variation was greater among $m e t 1$-epiRILs than among $d d m l$-epiRILs. There are several possible explanations for this: First, $d d m l$-epiRILs are derived from a backcross and should thus be generally less variable than metl-epiRILs, which are derived from F2 lines. Second, in contrast to the metl-epiRIls, the $d d m 1$ lines used in the crosses were already four generations old (Johannes et al. 2009). As some of the DNA methylation changes in $d d m 1$ mutants are known to revert over time (Johannes et al. 2009; Colome-Tatche et al. 2012), this may have contributed to the lower overall variability. Third, there is some indication that met 1 -epiRILs are less stable, with more frequent de novo epimutations than $d d m 1$ epiRILs (Johannes et al. 2009; Reinders et al. 2009; Colome-Tatche et al. 2012). Last, the two methylation mutants have different modes of action, with different degrees and patterns of methylation losses across the genome (Vongs et al. 1993; Kakutani et al. 1995; Saze et al. 2003; Lister et al. 2008; Stroud et al. 2013; Zemach et al. 2013), and this may also have contributed to the differences between the two epiRIL populations.

A particularly interesting finding is that the metl-epiRILs harbored three distinct life-history types which are also known from wild $A$. thaliana populations and are believed to reflect adaptation to different climates or habitats (Pigliucci and Marlow 2001; Pigliucci 2002). It is possible that the occurrence of these life-history types is associated with the inheritance of a demethylated FWA gene in met1epiRILs (Reinders et al. 2009). Indeed, from the subset of met 1 -epiRILs analysed by Reinders et al. 2009, all those with demethylated $F W A$ were late- or non-flowering types in our study (see Supplementary Figure S1). Although there is also some demethylation of $F W A$ in the $d d m l$ mutant (Stroud et al. 2013), this demethylation is much less widespread, and it does not appear to contribute much to variation in flowering time in these lines (Johannes et al. 2009; Cortijo et al. 2014). Of course, these ideas could only be verified through detailed molecular analyses, but if the different life-history types in metl-epiRILs are indeed epigenetically-based, then a single reshuffling between two epigenomes can re-create phenotypes similar to those shaped by long-term evolutionary forces (Ungerer et al. 2002; Stinchcombe et al. 2004).

One challenge in comparing epiRILs to RILs or natural ecotypes is that neither of these groups represents pure epigenetic or DNA sequence variation. Both epiRIL populations still harbor small amounts of DNA sequence variation (Johannes et al. 2009; Reinders et al. 2009): on the one hand a few SNPs-spontaneous mutations during epiRIL creation, or longer-term impurities of the wild-type inbred lines-and on the other hand sequence differences caused by activated transposable elements (TEs). These active TEs are consequences of the reduced DNA methylation which, among others, has the function to silence TEs in intact genomes. However, molecular analyses have shown that novel TE insertions in epiRILs are few, tend to occur randomly across the genome, and contribute little to phenotypic variation (Johannes et al. 2009; Reinders et al. 2009; Cortijo et al. 2014). Nevertheless, TE activity may have created some new sequence differences in the plants we worked with, and without high-resolution genomic data we cannot rule out that these may have contributed to the observed phenotypic variation among epiRILs.

Not only contain epiRILs some sequence variation, but of course RILs and natural ecotypes vary extensively also at the level of DNA methylation (Dubin et al. 2015; Schmitz et al. 2013b; Kawakatsu et al. 2016). Much of this epigenetic variation appears to be under genetic control though (e.g. Dubin et al. 2015). Notwithstanding the impurity of all studied lines, there remains a large qualitative difference between epiRILs and the other groups: in the epiRILs, epigenetic differences are several orders of magnitude larger than DNA sequence differences (Colome-Tatche et al. 2012), whereas in genetic RILs and natural ecotypes genetic and epigenetic differences are of the same order of magnitude. Hence, the critical assumption of our study is that the phenotypic differences observed among epiRILs are largely caused by underlying epigenetic differences (for $d d m l$ epiRILs this is supported by Cortijo et al. 2014), but among RILs and natural ecotypes they are mainly driven by sequence variation. Testing this assumption would require whole-genome and whole-methylome sequencing of the studied $\sim 200$ lines, and the statistical analysis of genotypeepigenotype-phenotype relationships through GWAS/ EWAS and (epi)QTL approaches.

In summary, we have employed quantitative genetic methods to compare the phenotypic variation in epiRILs of Arabidopsis thaliana with other groups of A. thaliana lines frequently used to study the genetic basis of phenotypic variation. We found that for some traits the phenotypic variation among epiRILs was comparable to that among RILs and natural ecotypes, and that epigenetic variation has the potential to create phenotypic variation that is stable and substantial, and thus of evolutionary significance. Future studies should aim to verify these findings through highresolution molecular and quantitative epigenetic analyses, and they should test the evolutionary significance of epigenetic variation also through experimental evolution approaches where genomic, epigenomic and phenotypic changes can be followed across multiple generations, and epigenetically-based evolution can thus be studied in action. 


\section{Data archiving}

All individual phenotype data available from the Dryad Digital Repository: https://doi.org/10.5061/dryad.b7bq47d

Acknowledgements We are grateful to Vincent Colot and Jurek Paszkowski for providing seed materials of epiRILs, and for their helpful suggestions on the manuscript. This work was supported by the Swiss National Science Foundation [grant no. 31EE30-131171 to O. B.] as part of the European Science Foundation EUROCORES Programme EuroEEFG, the National Natural Science Foundation of China [grant no. 31600291 to Y-Y.Z.], and the Fundamental Research Funds for the Central Universities in China [grant no. 20720170074 to Y-Y.Z.].

\section{Compliance with ethical standards}

Conflict of interest The authors declare that they have no conflict of interest.

\section{References}

Alonso-Blanco C, Koornneef M (2000) Naturally occurring variation in Arabidopsis: an underexploited resource for plant genetics. Trends Plant Sci 5:22-29

Becker C, Hagmann J, Muller J, Koenig D, Stegle O, Borgwardt K et al. (2011) Spontaneous epigenetic variation in the Arabidopsis thaliana methylome. Nature 480:245-249

Bird A (2007) Perceptions of epigenetics. Nature 447:396-398

Bossdorf O, Richards CL, Pigliucci M (2008) Epigenetics for ecologists. Ecol Lett 11:106-115

Brachi B, Villoutreix R, Faure N, Hautekeete N, Piquot Y, Pauwels M et al. (2013) Investigation of the geographical scale of adaptive phenological variation and its underlying genetics in Arabidopsis thaliana. Mol Ecol 22:4222-4240

Colome-Tatche M, Cortijo S, Wardenaar R, Morgado L, Lahouze B, Sarazin A et al. (2012) Features of the Arabidopsis recombination landscape resulting from the combined loss of sequence variation and DNA methylation. Proc Natl Acad Sci USA 109:16240-16245

Conner JK, Franks R, Stewart C (2003) Expression of additive genetic variances and covariances for wild radish floral traits: Comparison between field and greenhouse environments. Evolution 57:487-495

Cortijo S, Wardenaar R, Colomé-Tatché M, Gilly A, Etcheverry M, Labadie K et al. (2014) Mapping the epigenetic basis of complex traits. Science 343:1145-1148

Cubas P, Vincent C, Coen E (1999) An epigenetic mutation responsible for natural variation in floral symmetry. Nature 401:157-161

Dubin MJ, Zhang P, Meng D, Remigereau M-S, Osborne EJ, Casale FP et al. (2015) DNA methylation in Arabidopsis has a genetic basis and shows evidence of local adaptation. Elife 4:e05255

Falconer DS, Mackay TFC (1996) Introduction to quantitative genetics. Longman, New York, NY, USA

Finnegan EJ, Kovac KA (2000) Plant DNA methyltransferases. Plant Mol Biol 43:189-201

Fisher RA (1930) The genetical theory of natural selection. Oxford University Press, Oxford, UK

Futuyma DJ (2013) Evolution. Sinauer associates, Inc, Sunderland, Massachusetts, USA

Hansen TF, Pelabon C, Houle D (2011) Heritability is not evolvability. Evol Biol 38:258-277
Herrera CM, Bazaga P (2011) Untangling individual variation in natural populations: ecological, genetic and epigenetic correlates of long-term inequality in herbivory. Mol Ecol 20:1675-1688

Johannes F, Colot V, Jansen RC (2008) OPINION Epigenome dynamics: a quantitative genetics perspective. Nat Rev Genet 9:883-890

Johannes F, Porcher E, Teixeira FK, Saliba-Colombani V, Simon M, Agier $\mathrm{N}$ et al. (2009) Assessing the impact of transgenerational epigenetic variation on complex traits. PLoS Genet 5:e1000530

Kakutani T, Jeddeloh JA, Richards EJ (1995) Characterization of an Arabidopsis thaliana DNA hypomethylation mutant. Nucleic Acids Res 23:130-137

Kawakatsu T, Huang SSC, Jupe F, Sasaki E, Schmitz RJ, Urich MA et al. (2016) Epigenomic diversity in a global collection of Arabidopsis thaliana accessions. Cell 166:492-505

Kooke R, Johannes F, Wardenaar R, Becker F, Etcheverry M, Colot V et al. (2015) Epigenetic basis of morphological variation and phenotypic plasticity in Arabidopsis thaliana. Plant Cell 27:337-348

Koornneef M, Alonso-Blanco C, Vreugdenhil D (2004) Naturally occurring genetic variation in Arabidopsis thaliana. Annu Rev Plant Biol 55:141-172

Latzel V, Allan E, Silveira AB, Colot V, Fischer M, Bossdorf O (2013) Epigenetic diversity increases the productivity and stability of plant populations. Nat Commun 4:2875

Latzel V, Zhang Y, Moritz KK, Fischer M, Bossdorf O (2012) Epigenetic variation in plant responses to defence hormones. Ann Bot 110:1423-1428

Lister C, Dean C (1993) Recombinant inbred lines for mapping RFLP and phenotypic markers in Arabidopsis thaliana. Plant $\mathrm{J}$ 4:745-750

Lister R, O'Malley RC, Tonti-Filippini J, Gregory BD, Berry CC, Millar AH et al. (2008) Highly integrated single-base resolution maps of the epigenome in Arabidopsis. Cell 133:523-536

Loudet O, Chaillou S, Camilleri C, Bouchez D, Daniel-Vedele F (2002) Bay-0 x Shahdara recombinant inbred line population: a powerful tool for the genetic dissection of complex traits in Arabidopsis. Theor Appl Genet 104:1173-1184

Morgado L, Preite V, Oplaat C, Anava S, Ferreira de Carvalho J, Rechavi $O$ et al. (2017) Small RNAs reflect grandparental environments in apomictic dandelion. Mol Biol Evol 34:2035-2040

Ossowski S, Schneeberger K, Lucas-Lledo JI, Warthmann N, Clark RM, Shaw RG et al. (2010) The rate and molecular spectrum of spontaneous mutations in Arabidopsis thaliana. Science 327:92-94

Pigliucci M (2002) Ecology and evolutionary biology of Arabidopsis. Arab Book / Am Soc Plant Biol 1:e0003

Pigliucci M, Marlow ET (2001) Differentiation for flowering time and phenotypic integration in Arabidopsis thaliana in response to season length and vernalization. Oecologia 127:501-508

Pinheiro J, Bates D, DebRoy S, Sarkar D, R Core Team (2013). nlme: Linear and nonlinear mixed effects models. $\mathrm{R}$ package version 3.1-113, https://CRAN.R-project.org/package $=$ nlme

Platt A, Horton M, Huang YS, Li Y, Anastasio AE, Mulyati NW et al. (2010) The scale of population structure in Arabidopsis thaliana. PLoS Genet 6:e1000843

R Core Team (2013) R Foundation for Statistical Computing: Vienna, Austria.

Reinders J, Paszkowski J (2009) Unlocking the Arabidopsis epigenome. Epigenetics 4:557-563

Reinders J, Wulff BBH, Mirouze M, Mari-Ordonez A, Dapp M, Rozhon W et al. (2009) Compromised stability of DNA methylation and transposon immobilization in mosaic Arabidopsis epigenomes. Genes Dev 23:939-950 
Richards CL, Alonso C, Becker C, Bossdorf O, Bucher E, ColomeTatche $\mathrm{M}$ et al. (2017) Ecological plant epigenetics: evidence from model and non-model species, and the way forward. Ecol Lett 20:1576-1590

Richards EJ (2006) OPINION Inherited epigenetic variation - revisiting soft inheritance. Nat Rev Genet 7:395-401

Roux F, Colome-Tatche M, Edelist C, Wardenaar R, Guerche P, Hospital $\mathrm{F}$ et al. (2011) Genome-wide epigenetic perturbation jump-starts patterns of heritable variation found in nature. Genetics 188:1015-1017

Saze H, Scheid OM, Paszkowski J (2003) Maintenance of CpG methylation is essential for epigenetic inheritance during plant gametogenesis. Nat Genet 34:65-69

Schmitz RJ, He Y, Valdes-Lopez O, Khan SM, Joshi T, Urich MA et al. (2013a) Epigenome-wide inheritance of cytosine methylation variants in a recombinant inbred population. Genome Res 23:1663-1674

Schmitz RJ, Schultz MD, Lewsey MG, O’Malley RC, Urich MA, Libiger $\mathrm{O}$ et al. (2011) Transgenerational epigenetic instability is a source of novel methylation variants. Science 334:369-373

Schmitz RJ, Schultz MD, Urich MA, Nery JR, Pelizzola M, Libiger O et al. (2013b) Patterns of population epigenomic diversity. Nature 495:193-198

Simons AM, Roff DA (1994) The effect of environmental variability on the heritabilities of traits of a field cricket. Evolution 48:1637-1649

Stinchcombe JR, Weinig C, Ungerer M, Olsen KM, Mays C, Halldorsdottir SS et al. (2004) A latitudinal cline in flowering time in Arabidopsis thaliana modulated by the flowering time gene FRIGIDA. Proc Natl Acad Sci USA 101:4712-4717
Stroud H, Greenberg MVC, Feng SH, Bernatavichute YV, Jacobsen SE (2013) Comprehensive analysis of silencing mutants reveals complex regulation of the Arabidopsis methylome. Cell 152:352-364

Ungerer MC, Halldorsdottir SS, Modliszewski JL, Mackay TFC, Purugganan MD (2002) Quantitative trait loci for inflorescence development in Arabidopsis thaliana. Genetics 160:1133-1151

van der Graaf A, Wardenaar R, Neumann DA, Taudt A, Shaw RG, Jansen RC et al. (2015) Rate, spectrum, and evolutionary dynamics of spontaneous epimutations. Proc Natl Acad Sci USA 112:6676-6681

Vidalis A, Živković D, Wardenaar R, Roquis D, Tellier A, Johannes F (2016) Methylome evolution in plants. Genome Biol 17:264

Vongs A, Kakutani T, Martienssen RA, Richards EJ (1993) Arabidopsis thaliana DNA methylation mutants. Science 260:1926-1928

Wilschut RA, Oplaat C, Snoek LB, Kirschner J, Verhoeven KJF (2016) Natural epigenetic variation contributes to heritable flowering divergence in a widespread asexual dandelion lineage. Mol Ecol 25:1759-1768

Wolfe MD, Tonsor SJ (2014) Adaptation to spring heat and drought in northeastern Spanish Arabidopsis thaliana. New Phytol 201:323-334

Zemach A, Kim MY, Hsieh PH, Coleman-Derr D, Eshed-Williams L, Thao K et al. (2013) The Arabidopsis nucleosome remodeler DDM1 allows DNA methyltransferases to access H1-containing heterochromatin. Cell 153:193-205

Zhang Y-Y, Fischer M, Colot V, Bossdorf O (2013) Epigenetic variation creates potential for evolution of plant phenotypic plasticity. New Phytol 197:314-322 